\title{
A PROPOS DES CULICOIDES DE LA RÉGION BORDELAISE
}

\author{
Par A.-H. MANDOUL
}

J'ai signalé, dans ces Annales, la présence, dans la région bordelaise, d'un Culicoides piqueur, particulièrement agressif, le Culicoides perpungens Kieffer. Afin de préciser et d'étendre nos connaissances sur les Culicoides de notre région, j'avais indiqué à M. Meilhan, préparateur à la Station entomologique de la Faculté des Sciences, comme sujet de thèse, l'étude de ces diptères piqueurs. Appelé à se déplacer en raison de ses fonctions, M. Meilhan m'apparaissait tout désigné pour ce travail.

La moisson fut des plus fructueuses, trois nouvelles espèces vinrent se placer à côté du Culicoides perpungens : C. pulicaris L., C. obsoletus Meigen et $C$. albicans Winnertz. Dans cette énumération, ne figure pas l'espèce dont j'avais signalé la présence à Talence. La détermination de l'échantillon que j'avais capturé avait été faite par l'abbé Kieffer, bien connu par ses travaux et ses ouvrages sur les Chironomidés, enlevé depuis à la science, alors que ceux de M. Meilhan avaient été confiés à un savant spécialiste belge, M. Goetghebuer. Un désaccord entre ces deux spécialistes pouvait ètre à la base de ces différences d'interprétation.

Afin d'être fixé, j'ai adressé à M. Goetghebuer les échantillons sur lesquels l'abbé Kieffer avait fait sa détermination. La réponse fut : Culicoides albicans Winnertz. Il en résulte que le nombre des espèces trouvées dans la Gironde se réduit ainsi à trois. Il est à noter que $C$. perpungens ou albicans est de beaucoup le plus répandu aux environs de Bordeaux. Cette espèce est nouvelle pour la France, elle n'avait été signalée jusqu'ici qu'en Belgique, Rhénanie, Autriche, Hongrie.

Voici quelles sont les caractéristiques, d'après Kieffer, de ces trois espèces bordelaises, en les rangeant par ordre d'importance.

\section{Culicoïdes albicans Winnertz}

« Abdomen de la femelle blanchâtre, à pubescence blanche, celui du mâle brun, à poils blancs. Palpes jaunes à $2^{\circ}$ article très grossi, ellipsoïdal, égalant 3 et 4 réunis, ceux-ci égaux; panache brun à reflets d'or

Annales de Parasitologie, $\mathrm{T}$. VII, $\mathrm{N}^{\circ} 3 .-1^{\text {er }}$ mai 1929 , p. 218-221. 
ou, à l'extrémité, à reflets blancs. Antennes de la femelle blanchâtres, articles 10-14 ensemble à peine plus longs que 2-9 réunis, comme 51: 49 . Thorax gris, à poils rares et blancs, et, chez le mâlẹ, avec quelques poils noirs. Balanciers blancs, Aile blanche, les longues soies manquent chez le mâle dans les cellules sous-costale, médiale, radiale, dans la base de la cellule discoïdale postérieure et le long de la partie anté-

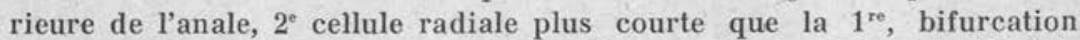
de $\mathrm{P}$ sous le milieu de la $1^{\text {ro }}$ cellule radiale, celle de $\mathrm{D}$ à peine distale de T. Pattes blanchâtres, extrémités des fémurs et des tibias noires, articles terminaux de la pince très amincis à l'extrémité, qui est recourbée en crochet, lamelle dorsale atteignant l'extrémité des articles basaux, avec une pointe à chaque angle postérieur. Longueur : mâle, $1 \mathrm{~mm} ., 4$, femelle, $1 \mathrm{~mm}$., 1. 》 (Kieffer, p. 78).

La larve est aquatique.

\section{Culicoïdes obsoletus Meigen}

Cette espèce a été signalée en France, en Allemagne, Autriche, Hollande et Angleterre.

* Aile de la femelle brunâtre, avec l'extrême base, l'extrémité et 2 bandes transversaies hyalines, la $1^{\text {re }}$ bande englobe la $1^{\text {re }}$ cellule radiale et $\mathrm{T}$, pour s'arrêter au milieu de la cellule anale; la $2^{\circ}$ commence derrière l'embouchure de $\mathrm{Cu}$ et atteint le bord postérieur au milieu de la cellule posticale; les soies longues seulement le long du bord depuis l'extrémité de Cu jusqu'à celles de P2 ; en outre, quelques rangées vont du milieu de la cellule cubitale et des 2 posticales jusqu'au bord ; bifurcation de $\mathrm{D}$ et de $\mathrm{P}$ sous le milieu de la $1^{\text {re }}$ cellule radiale; aile du mâle hyaline avec trois bandes transversales purpurines ou grises ; la $1^{\text {ro }}$ plus large, est proximale de $\mathrm{R}$ et s'arrête peu avant le bord postérieur ; les 2 autres sont percurrentes, l'une traverse la $2^{\circ}$ cellule radiale et la moitié distale de la $1^{\text {ro }}$ et englobe P2, l'autre, à égale distance de $\mathrm{Cu}$ et de la pointe alaire, aboutit à l'extrémité de PI, bifurcation de D sous le milieu de la $1^{\text {re }}$ cellule radiale, celle de $\mathrm{P}$ sous l'extrémité de cette cellule; quelques soies longues seulement le long du bord depuis $\mathrm{Cu}$ jusqu'à l'embouchure de $\mathrm{D} 2 ; 2^{\circ}$ celluke radiale plus courte que la $1^{\text {ro }}$ (mâle) ou l'égalant (femelle). Palpes jaunes, $2^{\circ}$ article grossi, ellipsoïdal, au moins aussi long que 3 et 4 réunis ; panache brunâtre, à reflets jaune d'or. Antennes de la femelle brunâtres, articles 10-14 einsemble aussi longs que 2-9 réunis. Thorax brun, à reflets gris sur le mésonotum, parfois avec une bande de chaque côté, sombre, raccourcie en avant. Balanciers blancs. Pattes blanc-jaunâtre, genoux noirs; articles terminaux de la 
pince grossis aux 2 bouts, mais plus à la base, lamelle dorsale tronquée, sans lobe. Longueur : mâle, 1 mm., 4, femelle, 1 mm., 1. » (Kieffer, p. 82).

\section{Culicoïdes pulicaris Linne}

C'est l'espèce la plus anciennement connue, la plus répandue, elle est signalée à peu près dans toute l'Europe.

«Cellnle posticale à soies longues sur toute sa surface, des soies pareilles sur toute l'aile de la femelle, sauf entre C (nervure costale) d'une part, et Sc (sous-costale), R et Cu d'autre part, entre Sc et M (nervure médiane), entre $\mathrm{M}$ et $\mathrm{P}$, entre $\mathrm{P}$ et $\mathrm{A}$ (nervure anale); l'aile du mâle n'a des soies longues que dans la cellule cubitale, les 2 discoïdales et la posticale; base à lobe rectangulaire, surface blanche avec de petites täches noires nombreuses, dont une au centre de la cellule posticale, une à l'extrémité des discoïdales antérieure et postérieure, les autres formant 3 rangées transversales, dont la $1^{\text {re }}$, un peu distale du milieu de $\mathrm{Sc}$, comprend une tache unissant $\mathrm{C}$ à $\mathrm{M}$, et une sur $\mathrm{A}$; la $2^{\circ}$ avec une sur $\mathrm{C}$ et la jonction des 2 cellules radiales, une sur la base de D2 et une sur la base de P2; la $3^{\circ}$ avec une grande tache en sablier dans la cellule cubitale, une dans la partie distale de chacune des deuxièmes cellules discoïdales; deuxième cellule radiale plus courte que la $1^{\text {re }}$; tige de $\mathrm{D}$ égalant $\mathrm{T}$, bifurcation de $\mathrm{P}$ à peine distale de celle de D : Brun noir. Yeux amincis en haut et se touchant par un point; $2^{\circ}$ article des palpes pas plus long que le premier, égalant 3 et 4 réunis, grossi fortement au milieu du côté médial où se trouve l'organe sensoriel, entouré de poils en massue, panache fauve à reflets jaunes. Antennes: articles 12-14 ensemble plus courts que 2-11 réunis, comme $63 ; 67 ; 10-14$, comme $7,5: 8,5: 21,5 ; 20 ; 21,5$, articles $10-14$ de la femelle subcylindriques ensemble à peine plus longs que 2-9 réunis, $10^{\circ}$ moitié plus long que le $9^{\circ}$, qui est deux fois aussi long que gros. Mésonotum gris, souvent en avant avec une grande tache brune en losange, ayant les angles rayonnants, les latéraux reliés à une tache latérale, parfois seulement à taches isolées, ou sans trace de tache. Balanciers blancs. Pattes brunes, articulation des tarses souvent noirâtres; articles terminaux de la pince amincis dans la moitié distale, lamelle dorsale à 2 petits lobes pointus et, entre ceux-ci, 2 petits appendices velus. Longueur : mâle et femelle, $1 \mathrm{~mm}$., 5 à $2 \mathrm{~mm}$., 5 . 》 (Kieffer, p. 87).

La larve est aquatique et vit dans les eaux stagnantes. 


\section{RÉSumÉ}

L'espèce bordelaise de Culicoides dont j'avais signalé la présence, sous le nom de Culicoides perpungens, d'après la détermination de Kieffer, serait pour Goetghebuer le C. albicans.

On doit ajouter à la liste de ces diptères, trouvés dans les environs de Bordeaux, deux nouvelles espèces capturées par Meilhan : Culicoides obsoletus et $C$. pulicaris. Le $C$. albicans est de beaucoup le plus répandu.

\section{BiBLIOGRAPHIE}

Kieffer. - Chironomide Ceratopogonina. Faune de France, Paris, Lechevalier, 1925.

Goztghenuer (M.). - Ceratopogonines de Belgique. Mém. Musée royal d'hist. nat. de Belgique, 1920.

Mandoul (A.-H.). - Un nouveau diptère piqueur de la région bordelaise. Journ, de Méd. de Bordeaux, 25 juin 1926.

- Présence à Bordeaux de Culicoïdes perpungens. Ann. de parasitologie, IV, 1926 , p. 319-322.

Metlhan. - Contribution à l'étude des Culicoïdes de la région bordelaise. Thèse de Bordeaux, 1927. 